\title{
B-Glucuronidase as a new potent biomarker in the diagnosis and prognosis of organophosphorus toxicity
}

\author{
Bhagwat Kale \\ Department of Biochemistry, Solapur Institute of Paramedical Science, \\ Civil Lines, Near Rangbhavan, Opposite to District Court, Solapur - 413004. \\ Email: kalebhagwat2644@ rediffmail.com
}

\begin{abstract}
Background: Organophosphorus poisoning occurs as a result of accidental exposure, suicidal and homicidal attempts. Organophosphorus compounds are known to cause selective release of liver microsomal $ß$-glucuronidase (BG) into serum. Dissociation of the egasyn - $\beta$-glucuronidase complex in the liver by organophosphorus compounds was followed by a massive release of $\beta$-glucuronidase from liver into the serum.

Objectives: There are few papers suggesting increase in activity of B-glucuronidase (BG) in serum of experimental animals subjected to organophosphorus poisoning. But, still there is less or no such study in human beings having organophosphorus poisoning. Thus, aim of our this research was to estimate and correlate activity of ß-glucuronidase (BG) in different stages of organophosphorus poisoning.

Methods: B-glucuronidase (BG) activity was determined by Goldstein method.

Results and Conclusions: We found that the serum activity of $\beta$ - glucuronidase (BG) proportionally increases $(\mathrm{p}<0.01)$ and correlates with severity of organophosphorus poisoning. Further, there was found a significant increase in $\beta$-glucuronidase (BG) activity in low dose (mild) poisoning also. Thus, $\beta$ - glucuronidase (BG) can be used as a potent biochemical marker in the diagnosis and prognosis of organophosphorus poisoning along with acetylcholinesterase or may also without acetylcholinesterase.
\end{abstract}

Keywords: acetylcholinesterase, delayed polyneuropathy, $\beta$-glucuronidase, intermediate syndrome and organophosphorus.

\section{Introduction}

A pesticide is usually defined as a chemical substance, biological agent, antimicrobial agent or disinfectant used against pests including insects, plant pathogens, weeds, molluscs, birds, mammals, fish, nematodes (roundworms) and microbes that compete with humans for food, destroy property, have a propensity for spreading or are a vector for disease or simply a nuisance. The term insecticide is used to denote agents designed to kill only insects, but the term pesticide has a broader meaning and also includes herbicides, rodenticides, fumigants, nematocides, algaecides, ascaricides, molluscicides, disinfectants, defoliants and fungicides.[1] Pesticide poisonings remain a serious public health problem worldwide. According to the World Health Organization's estimate, 30,00,000 cases of pesticide poisoning occur every year, resulting in more than 2,50,000 deaths.[2,3] Among the numerous pesticides that can result in death, organophosphate insecticides are the most common culprit agents because of their high toxicity.[4]

Organophosphate (OP) insecticides are potent inhibitors of acetylcholinesterase enzyme. This inhibition leads to an increase in acetylcholine activity, which is responsible for symptoms seen in organophosphorus poisoning such as abdominal pain, diarrhea, hyper-sialorrhea, tight myosis and vomiting.[5,6] The primary mechanism of action of organophosphorus pesticides is based on inhibition of the acetylcholinesterase (AChE) enzyme. Once acetylcholinesterase has been inactivated, acetylcholine (ACh) accumulates throughout the nervous system, resulting in overstimulation of muscarinic and nicotinic receptors [7,8].

Overstimulation of these receptors are associated with severe symptoms of poisoning. Level of acetylcholinesterase inhibition and severity of poisoning symptoms in humans increases with increased pesticide doses, exposure time, atmospheric temperature, developmental age and sex [9]. Thus, a progressive decrease in acetylcholinesterase activity is proportional to increase in severity of poisoning which reflects proportionate amount of pesticide consumed and absorbed in the body [10].

On the basis of severity of clinical symptoms, organophosphorus poisoned patients are divided into three stages as mildly poisoned, moderately poisoned and severely poisoned [11]. 
1) Mild poisoning stage: It is initial stage of organophosphorus poisoning and is acute cholinergic crisis. This stage is associated with number of poisoning symptoms such as muscle twitching, headache, dizziness, tremor, restlessness, anxiety, confusion, convulsions and coma. Patients can also develop pancreatitis, hypo or hyperglycemia and acute renal failure during this phase. This is also called as type - I paralysis and it generally occurs in the first 48 hours of poisoning $[12,13]$.

2) Moderate poisoning stage: It is the stage associated with intermediate syndrome. It is type - II paralysis. It occurs between 48 to 72 hours after poisoning. Symptoms observed in this stage include weakness of neck flexors, muscles of respiration and proximal limb muscles. Intermediate syndrome is a state of muscle paralysis that occurs after recovery from cholinergic crisis but before the expected onset of the delayed polyneuropathy and probably results from post-synaptic neuromuscular junction dysfunction.

According to De Bleecker et.al., the slow release of organophosphorus compounds from deep tissue and persistent inhibition of acetylcholinesterase are responsible for development of intermediate syndrome. According to Mathew et.al. intermediate syndrome is due to severe muscle damage in organophosphorus poisoning patients and the magnitude of muscle damage that occurs during cholinergic crisis determines the severity of intermediate syndrome. Intermediate syndrome requires prolonged mechanical ventilation and chances of mortality increases in this stage $[13,14]$.

3) Severe poisoning stage: It is the severe stage associated with delayed polyneuropathy. It is type - III paralysis. It occurs 15 to 30 days after exposure to the organophosphorus poisoning. Delayed polyneuropathy occurs due to inhibition of Neuropathy Target Esterase (NTE) an enzyme present in nervous tissue. Clinical manifestations of this stage are distal weakness, parasthesia, ataxia and diminished reflexes. Mortality rate is very high in this stage. [15,16]

N. Senanayke, H J de silva and L. Karalliedde in 1993 developed a scoring system to access severity of poisoning. This scoring system is the Peradeniya Organophosphorus Poisoning (POP) scale. In this scale, common clinical manifestations of organophosphorus poisoning are selected as parameters and each is assessed on a three points scale varying from 0 to 2.[17] (Table 1)

Table1: Peradeniya Organophosphorus Poisoning (OPP) scale.

\begin{tabular}{|c|c|c|}
\hline Parameter & Clinical Criteria & Score \\
\hline \multirow{3}{*}{ Pupil size } & $>2 \mathrm{~mm}$ & 0 \\
\hline & $<2 \mathrm{~mm}$ & 1 \\
\hline & Pin - Point & 2 \\
\hline \multirow{3}{*}{ Respiratory rate } & $<20 / \min$ & 0 \\
\hline & $>20 / \min$ & 1 \\
\hline & $>20 /$ min with central cyanosis & 2 \\
\hline \multirow{3}{*}{ Heart rate } & $>60 / \min$ & 0 \\
\hline & $41-60 / \min$ & 1 \\
\hline & $<40 / \mathrm{min}$ & 2 \\
\hline \multirow{3}{*}{ Fasciculation } & None & 0 \\
\hline & Present, generalized or continuous & 1 \\
\hline & Both, generalized and continuous & 2 \\
\hline \multirow{3}{*}{ Level of Consciousness } & Conscious and rational & 0 \\
\hline & Impaired response to verbal commands & 1 \\
\hline & No response to verbal commands & 2 \\
\hline \multirow{2}{*}{ Seizures } & Absent & 0 \\
\hline & Present & 1 \\
\hline
\end{tabular}

The score is obtained at initial presentation before any medical treatment and it represents the muscarinic, nicotinic and central effects of the acute cholinergic manifestations of organophosphorus poisoning. A score of 0 to 3 is considered as mild poisoning, 4 to 7 as moderate poisoning and 8 to 11 as severe poisoning [18].

Acute toxicity of organophosphorus poisoning induces severe hepatic damage. This leads to increased levels of aspartate transminase (AST), alanine transminase (ALT) and $\gamma$-glutamyl transpeptidase (GGT) which represents marked 
hepatic tissue injury [19]. The ratio of lactate/ pyruvate is increased in the liver which is referred to as an enhancement of anaerobic glycolysis. The increased level of the serum lactate is observed which is due to tissue hypoxia [20].

According to TOS-Luty et.al. an organophosphorus compound malathion induces damages in the intercellular structure of the liver [21]. Slizova et.al. further stated that organophosphorus poisoning causes changes in the microvascularization of liver [22] Further, serum total protein level is decreased due to lowered synthesis of albumin in liver in organophosphorus poisoning. Serum globulins (mostly $\alpha$-globulin) rises due to prolonged exposures to organophosphorus compounds [23].

In experimental models, organophosphorus compounds are known to cause selective release of liver microsomal ß-glucuronidase (BG) into serum. Egasyn is an accessory protein of B-glucuronidase in the liver microsomes. Liver microsomal B-glucuronidase is stabilized within the luminal site of microsomal vesicles by binding to egasyn, which is one of the carboxyl esterase isozyme. Dissociation of the egasyn- $\beta$-glucuronidase complex by organophosphorus compounds in vivo was followed by a massive release of $\beta$-glucuronidase into the serum. There are only a few studies made on the changes of blood B-glucuronidase activity as a biomarker in acute organophosphorus compounds poisoning in animals $[24,25,26]$.

Acetylcholinesterase is already well known marker for organophosphorus poisoning in human beings. But, our interest was to known for another new marker. There are few papers suggesting increase in activity of $\beta$-glucuronidase (BG) in serum of experimental animals subjected to organophosphorus poisoning. But, still there is no such study in human beings having organophosphorus poisoning. Thus, aim of our this research was to estimate and correlate activity of B-glucuronidase $(\mathrm{BG})$ in different stages of organophosphorus poisoning.

\section{Materials and methods}

\subsection{Subjects}

The study was conducted at Department of Biochemistry, V. G. Shivdare College of Biotechnology, Solapur during the period October - 2010 to December -2011. We have studied total 50 organophosphorus poisoned patients and 50 controls. Organophosphorus poisoned patients were identified by attending physicians on the basis of symptoms shown by patients. Symptoms such as hyper-salivation, convulsions, respiratory failure, ataxia, slurred speech, miosis, muscle cramping suggest about organophosphorus poisoning. However, to access organophosphorus poisoning, it is necessary to analyze biological samples mostly blood and/or urine. Organophosphorus poisoning was confirmed by noting decreased acetyl cholinesterase activity. Organophosphorus poisoned patients were categorized into three groups as mildly poisoned patients, moderately poisoned patients and severely poisoned patients on the basis of symptoms shown by the patients and Peradeniya Organophosphorus Poisoning (POP) score illustrated in Table. (Table 2)

Table -2: Distribution of patients according to severity of poisoning on the basis of WHOs criteria

\begin{tabular}{lllc}
\hline $\begin{array}{l}\text { Grade of } \\
\text { poisoning }\end{array}$ & Symptoms & POP Score & No. of cases \\
\hline Mild & Nausea, vomiting, diarrhea, sweating etc. & 0 to 3 & 15 \\
\hline Moderate & $\begin{array}{l}\text { Lacrimation, salivation, miosis, } \\
\text { fasciculation etc. }\end{array}$ & 4 to 7 & 22 \\
\hline Severe & Incontinence, apnoeic spells, ARDS, areflexia, seizures, coma etc. & 8 to 11 & 13 \\
\hline
\end{tabular}

\subsection{Collection of samples}

Blood samples of organophosphorus poisoned patients were collected from ICU of Shri. Chhatrapati Shivaji Maharaj General Hospital, Solapur at the time of admission and before treatment with atropine plus PAM. 50 samples of controls were collected from Damani Blood Bank. Patients with any other type of poisoning than organophosphorus poisoning were excluded from the study group. Individuals with any disease/s and similarly, sprayers and individuals working in the vicinity of different pesticides and chemical industries were excluded from the study.

Total $5 \mathrm{ml}$ of venous blood were collected in a plain bulb. The obtained blood was centrifuged at $3000 \mathrm{rpm}$ for 10 minutes to obtain serum and cells. The analysis was done immediately by using separated serum.

\subsection{Estimation of $\beta$ - glucuronidase (BG) activity}

Serum $\beta$-glucuronidase activity was determined by Goldstein method. $\beta$-glucuronidase acts on the substrate, phenolphthalein mono $\beta$-glucuronide and cleave it into phenolphthalein and glucuronic acid. Phenolphthalein released produces pink color in an alkaline medium. Intensity of this pink color is proportional to activity of $\beta$ - glucuronidase. 
In two test-tubes, $0.6 \mathrm{ml}$ serum were taken and $4.8 \mathrm{ml}$ of $0.1 \mathrm{M}$ acetate buffer, $\mathrm{pH} 4.5$ were added. Then, $0.6 \mathrm{ml}$ of substrate was added to the one tube (Test). Both tubes were stopperred and kept in a water-bath at $37^{\circ} \mathrm{C}$ for 24 hours. On next day, tubes were removed $0.6 \mathrm{ml}$ substrate was added to the other tube (Control/Blank). In third tube (Standard), $0.6 \mathrm{ml}$ phenolphthalein standard, $4.8 \mathrm{ml} 0.1 \mathrm{M}$ acetate buffer, $\mathrm{pH} 4.5$ and $0.6 \mathrm{ml}$ distilled water were taken. The color produced was measured at $540 \mathrm{~nm}$ [27].

\section{Results and Discussion}

A present study includes 50 organophosphorus poisoned patients in which 15 were mild, 22 were moderate and 13 were severe. This distribution of organophosphorus poisoned patients into three grades as mildly poisoned patients, moderately poisoned patients and severely poisoned patients were done according to criteria of World Health Organization (WHO). This distribution were done on the basis of symptoms of organophosphorus poisoning and Peradeniya Organophosphorus Poisoning (POP) score. All the organophosphorus poisoned patients included in this study were belongs to age range in between 11 to 70 years. We studied total 50 patients from which 32 were males and 18 were females. (Table 3 )

Table 3: Mode of poisoning

\begin{tabular}{llll}
\hline Mode of poisoning & Males & Females & No. of cases \\
\hline Suicidal attempts & 23 & 14 & 37 \\
\hline Accidental poisoning & 09 & 04 & 13 \\
\hline Total & 32 & 18 & 50 \\
\hline
\end{tabular}

Number of males were found slightly higher than females because they have more economic burden of family in villages as compare to urban sides. Organophosphorus poisoning remains one of the major health issue in developed and especially in developing countries and its frequency is increasing over the years. In our study, 37 cases of poisoning found as a result of suicidal attempts while 13 due to accidental events.(Table 3 ) It is found that organophosphorus pesticide, dimethoate is more commonly used to attempt suicide as it is easily available. Majority of poisoning cases were due to diamethoate. The other cases of poisoning were due to sumethrine, parathion, deltanuthrin, malathion, cypermethrin, chlorpyriphos, tick - 20 etc. Out of 50 patients studied by us, 39 gets cured while 11 patients expired that includes 3 females. The death generally occurred due to respiratory failure. The results were analyzed by using Tukey test for multiple comparison of $\mathrm{k}$ population means (unequal sample sizes). 'p' value $<0.01$ were considered significant. The results are summarized as follows. (Table 4)

Table 4: Activities of $\beta$-glucuronidase (BG) in controls and organophosphorus poisoned patients.

\begin{tabular}{lllll}
\hline Parameters & Control & Mild & Moderate & Severe \\
\hline No. of cases (n) & $\mathrm{n}=50$ & $\mathrm{n}=15$ & $\mathrm{n}=22$ & $\mathrm{n}=13$ \\
\hline $\boldsymbol{\beta}$ - glucuronidase (BG) activity & 3349.46 & 4242.00 & 4878.59 & 5659.23 \\
in U/L (Mean \pm SD) & \pm & \pm & \pm & \pm \\
& 58.97 & 72.50 & 88.82 & 71.80 \\
\hline 'p' value when compared with control & $-\cdots---$ & $\mathrm{p}<0.01$ & $\mathrm{p}<0.01$ & $\mathrm{p}<0.01$ \\
\hline
\end{tabular}

Enzymatic status in mildly poisoned patients (Group I) : As compared to healthy controls, the activity of $\beta$ - glucuronidase were significantly increased $(\mathbf{p}<\mathbf{0 . 0 1})$ in mildly poisoned patients (Group I). $\beta$ - glucuronidase activity were $\mathbf{1 . 2 3}$ times higher in mildly poisoned patients as compared to those in healthy controls. (Table 4)

Enzymatic status in moderately poisoned patients (Group II) : As compared to healthy controls and mildly poisoned patients (group I) the activity of $\beta$ - glucuronidase were significantly elevated $(\mathbf{p}<\mathbf{0 . 0 1})$ in moderately poisoned patients (group II). $\beta$ - glucuronidase activity in group II patients were found increased 1.46 times and 1.15 times as compared to healthy controls and group I patients respectively. (Table 4)

Enzymatic status in severely poisoned patients (Group III) : As compared to healthy controls and moderately poisoned patients (Group II), the activity of $\beta$-glucuronidase were significantly increased (p $<\mathbf{0 . 0 1 )}$ in severely poisoned patients (Group III). In severely poisoned patients the activity of $\beta$-glucuronidase were $\mathbf{1 . 6 9}$ times higher as compared to controls and $\mathbf{1 . 1 6}$ times higher as compared to group II patients. (Table 4)

Thus, we found a proportional increase in $\beta$-glucuronidase (BG) activity with severity of organophosphorus poisoning which will helpful in the diagnosis and prognosis of organophosphorus poisoning . 


\section{Conclusion}

In this study, we found that the serum activity of $\beta$ - glucuronidase (BG) proportionally increases and correlates with severity of organophosphorus poisoning. Further, there was found a significant increase in $\beta$ - glucuronidase (BG) activity in low dose (mild) poisoning also. Thus, $\beta$ - glucuronidase (BG) can be used as a potent biochemical marker in the diagnosis and prognosis of organophosphorus poisoning along with acetylcholinesterase or may also without acetylcholinesterase.

\section{Acknowledgment}

I would like to thank the Biochemistry Department of V. G. Shivdare College, Solapur. I am also indebted to all the patients and my colleagues for their co-operation in this research.

\section{References}

[1] T Satoh. Release of liver microsomal beta-glucuronidase from hepatocytes in vitro and in vivo by organophosphates and hepatotoxic agents. J Toxicol Sci ; 1 (1991) : 133-142.

[2] T Satoh, S Suzuki, N Kawai, T Nakamura, M Hosokawa. Toxicological significance in the cleavage of esterase-beta-glucuronidase complex in liver microsomes by organophosphorus compounds. Chem Biol Interact; 119 (1999) : 471-478.

[3] SH Inayat-Hussain, SH Lubis, NI Sakian et al. Is plasma beta-glucuronidase a novel human biomarker for monitoring anticholinesterase pesticides exposure? A Malaysian experience. Toxicol Appl Pharmacol; 219 (2007) : 210-216.

[4] H J Mason. The recovery of plasma cholinesterase and erythrocyte acetylcholinesterase activity in workers after overexposure to dichlorvos. Occup. Med (Lond.) ; 50 (2000) : 343-347.

[5] C Roshini, J Visalakshi. Biochemical and clinical profile after organophosphorus poisoning-A plasebo-controlled trial using pralidoxime. J Assoc Physicians India;53 (2005):422-4.

[6] Y Fujikawa, T Satoh, S Suganuma et al. Extremely sensitive biomarker of acute organophosphorus insecticide exposure. Hum Exp Toxicol; 24 (2005) : 333-336.

[7] J Patil, A Patil et.al. Biochemical effects of various pesticides on sprayers of grape gardens. Ind J Clin Biochem; 18/2 (2003):16-22.

[8] P Lee, DYH Tai. Clinical features of patients with acute organophosphate poisoning requiring intensive care. Intensive Care Med;27 (2001) :694-9.

[9] S Khan, R Hemalatha, L Jeyaseelan, A Oommen, A Zachariah. Neuroparalysis and oxime efficacy in organophosphate poisoning: a study of butyrylcholinesterase. Hum Exp Toxicol; 20 (2001):169-74.

[10] K Kandalaft, S Liu, C Manivel et al. Organophosphate increases the sensitivity of human exocrine pancreas to acetylcholine. Pancreas; 6 (1991) : 398-403.

[11] RL Goodale, JC Manivel, JW Borner et al. Organophosphate sensitizes the human pancreas to acinar cell injury: an ultrastructural study. Pancreas; 8 (1993) : 171-5.

[12] RM Hassan, AJ Pesce, P Sheng, IB Hanenson. Correlation of serum pseudocholinesterase and clinical course in two patients poisoned with organophosphate insecticides. Clin Toxicol;18/4 (1981): :401-6.

[13] N Brahmi, A Mokline, N Kouraichi et al. Prognostic value of human erythrocyte acetylcholinesterase in acute organophosphate poisoning. Am J Emerg Med , 24/7 (2006) :822-7.

[14] B Sharma, S Bano et.al. Human acetyl cholinesterase inhibition by pesticide exposure. J Chinese Clin Med; 4 /1 (2009): 55-60.

[15] J Vidyasagar, N Karunakar, D Krishna et.al. Oxidative stress and antioxidant status in acute organophosphorus insecticide poisoning. Ind J Pharmacol; 36/2 (2004): $76-9$.

[16] O Sakrak, O Ekinci et.al. Effects of acute fenthion toxicity on liver and kidney function and histology in rats. Turk J Med Sci; 37/5 (2007) :281-8.

[17] A Hussain, J Jakoniux et.al. Organophosphate insecticide chlorfenvinphos affects enzymatic and non-enzymatic antioxidants in erythrocytes and serum of rats. Polish J Env Studies; $12 / 4$ (2003) :417-23.

[18] M Dandapani, L Jeyaseelan, A Oommen et.al. Oxidative damage in intermediate syndrome of acute organophosphorus poisoning. Ind J Med Res; 117 (2003) :253-59.

[19] J Bajgar. Complex view on poisoning with nerve agents and organophosphates. Acta Medica; 48/1 (2005):3 - 21.

[20] B Venkataraman, P Pais, T Joseph et.al. Correlation between the severity of symptoms in organophosphorus poisoning and cholinesterase activity (RBC and plasma) in humans. Ind J Physiol Pharmacol; 40/3 (1996) : 249-52.

[21] S Rastogi, P Satyanarayan, D Ravishankar, S Tripathi. Oxidative A study on oxidative stress and antioxidant status of agricultural workers exposed to organophosphorus insecticides during spraying. Ind J Occup Env Med; 13/3 (2009): 131-134.

[22] M Sungur, M Guven. Intensive care management of organophosphate insecticide poisoning. Critical care; $5 / 4$ (2001):211-5.

[23] B Paudyal. Organophosphorus poisoning. J Nepal Med Asso; 47/172 (2008) :251-8.

[24] T Leibson, M Lifshitz et.al. Organophosphate and carbamate poisoning : review of the current literature and summary of clinical and laboratory experience in southern Israel. IMAJ; 10 (2008) :767-70.

[25] E Reiner. Organophosphorus compounds and Esterases : Current research topics concerning toxicity of and protection against organophosphates. Institute for Med Research and Occupational Health Croatia (2001):1-7.

[26] S Rehiman, S Lohani, M Bhattarai et.al Correlation of serum cholinesterase level, clinical score at presentation and severity of organophosphorus poisoning. J Nepal Med Asso; 47/170 (2008):47-52.

[27] G Goldstein. Serum beta-glucuronidase assay by the phenolphthalein mono-beta-glucuronide method. Clin Chem; 7 (1961) : 136-142. 